\title{
PROGRESSO TEMPORAL DA FERRUGEM E FUNGICIDAS PARA CONTROLE DAS DOENÇAS FOLIARES DO PESSEGUEIRO $^{1}$
}

\author{
GISELDAALVES², GABRIEL MARTINS FERREIRA ${ }^{3}$ LOUISE LARISSA MAY DE MIO ${ }^{4}$
}

RESUMO - O objetivo do trabalho foi avaliar o progresso da ferrugem do pessegueiro e a eficiência de fungicidas no controle de da ferrugem e do furo-de-bala em pomares da região metropolitana de Curitiba-PR. Os ensaios foram conduzidos em pomar comercial com o delineamento experimental em blocos ao acaso, Cultivar Chimarrita, com quatro tratamentos (mancozebe, tiofanato metílico, clorotalonil e testemunha, sem pulverização) e quatro repetições, nas safras de 2004/2005 e 2005/2006. Cada fungicida foi pulverizado oito vezes, com intervalo de 15 a 20 dias entre as aplicações. A avaliação do progresso da epidemia de ferrugem e a eficiência dos fungicidas no controle das doenças foram feitas com base na incidência e severidade da doença nas folhas e na porcentagem de desfolha das plantas. A taxa de progresso da doença e o inóculo inicial na testemunha não diferiram significativamente entre os anos. Todos os fungicidas reduziram a desfolha e a severidade da ferrugem, mas o clorotalonil foi melhor e também reduziu a incidência em ambos os anos. Os fungicidas mancozebe e tiofanato metílico reduziram a incidência de furo-de-bala.

Termos para indexação: Prunus persica, Tranzschelia discolor, Wilsonomyces carpophilus incidência, controle químico.

\section{TEMPORAL PROGRESS OF PEACH RUST AND FUNGICIDES TO CONTROL THE FOLIAR DISEASES OF PEACH TREE}

\begin{abstract}
The aim of this study was to evaluate the development of the peach rust in two seasons and, the efficiency of fungicides to control the peach rust and the shot-hole in orchards of the metropolitan region of Curitiba, Paraná, Brazil. The trials were carried out in a commercial orchard and were designed in complete randomized blocks, cv. Chimarrita, with four treatments (mancozeb, thiophanate methyl, chlorothalonil and control-unsprayed) and four replications, in the crop seasons 20004/5 and 2005/06. Each fungicide was sprayed eight times, at 15 to 20 days intervals. The assessment of the epidemic progress and effectiveness of fungicide were performed based on the incidence and severity on leaves and percentage of plants defoliation. The progress rate of the epidemic and the initial inoculum did not differ significantly between years and the logistic model fit to data of both years. All fungicides reduced the defoliation and severity of peach rust, but chlorothalonil was the best and also reduced the incidence in both years. The fungicides mancozeb and thiophanate methyl reduced the shot-hole incidence.
\end{abstract}

Termos para indexação: Prunus persica, Transzchelia discolor, Wilsonomyces carpophilus incidence, chemical control.

\section{INTRODUÇÃO}

A ferrugem (Tranzschelia discolor (Fuckel) Tranzschel \& Litv.) e furo-de-bala (Wilsonomyces carpophilus (Lév.) Adaskaveg, Ogawa \& Butler) são as principais doenças foliares do pessegueiro. Regiões com temperatura e umidade elevadas, frequentes nas áreas subtropicais brasileiras entre os meses de setembro a abril, favorecem o aumento expressivo dessas doenças, tornando-as importantes em várias regiões produtoras de pêssego.

O pessegueiro, por ser uma planta decídua, começa a perder as folhas a partir de abril; entretanto, em pomares com elevado grau de severidade de ferrugem (cerca de 15\% da área foliar tomada pela doença), a planta, em uma ação de defesa, perde a folhagem antes da época prevista. Essa queda antecipada das folhas afeta o equilíbrio fisiológi-

\footnotetext{
${ }^{1}$ Trabalho Sinfruit 060 - Simpósio Internacional de Fruticultura - Avanços na Fruticultura (17 a 21 Outubro) ${ }^{2}$ Eng. Agr., Doutoranda Produção Vegetal/UFPR, Curitiba-PR. 84030-900. E-mail giseldaalves@uol.com.br ${ }^{3}$ Eng. Agr., Mestrando Produção Vegetal /UFPR, Curitiba-PR,84030-900. E-mail: gabrielmartins_agro@hotmail.com.

${ }^{4}$ Prof Dra Departamento de fitotecnia e fitossanitarismo / Produção Vegetal/UFPR, Curitiba-PR,84030-900. E-mail: maydemio@ufpr.br
} 
co, podendo proporcionar florações desuniformes, esgotando as reservas nutricionais, prejudicando a produtividade do pomar (ALVES et al., 2008; ALVES; MAY-DE MIO, 2008). Os sintomas do furo-de-bala ocorrem nas folhas que apresentam lesões avermelhadas, cujo centro se destaca, ficando a folha toda perfurada, normalmente a partir do final de agosto, atingindo o máximo da doença no mês de outubro (CHALLIOL et al., 2006). Seu prejuízo é resultado pela diminuição da área foliar fotossinteticamente ativa, com consequente redução da produção.

Os danos causados por essas doenças nas áreas produtoras de pêssego do Estado do Paraná têm exigido uso frequente de fungicidas, devido à suscetibilidade apresentada pelas cultivares plantadas. No Paraná, apesar de os fungicidas estarem cadastrados para a cultura, poucas informações estão disponíveis sobre o controle de doenças foliares. Além disso, não se conhece o comportamento da ferrugem, principal doença foliar, entre os municípios da região metropolitana. De acordo com o exposto acima, conduziu-se este trabalho para analisar o progresso da ferrugem em pomares de dois municípios deste estado e testar a eficiência de fungicidas no controle da ferrugem e do furo-de-bala do pessegueiro.

\section{MATERIAL E METODOS}

A avaliação de fungicidas no controle da ferrugem foi realizada em 2004/2005 e 2005/2006, em pomares comerciais de pessegueiro cv. 'Chimarrita', localizados nos municípios de Campo Largo e Araucária, principais regiões produtoras de pêssego no Estado do Paraná. Em Araucária, a área experimental foi de $2.300 \mathrm{~m}^{2}$ e em Campo Largo foi de aproximadamente $3.000 \mathrm{~m}^{2}$. O experimento com furo-de-bala foi realizado somente em Araucária.

O delineamento experimental foi o de blocos casualizados, com quatro tratamentos dos seguintes ingredientes ativos, com as respectivas doses, para cada $100 \mathrm{~L}$ de água: mancozeb (160 g i.a.), tiofanato metílico (51 g i.a.), clorotalonil (127,5 g i.a.) e a testemunha (sem pulverização). Cada tratamento contou com quatro repetições, e cada parcela, com três plantas, sendo a planta central considerada útil. As pulverizações foram realizadas com pulverizador costal e ação motorizada da marca Yamaha, modelo LS-937, que apresenta princípio de funcionamento mecânico, bico do tipo cônico, homogeneizador hidráulico e pressão sobre o produto em depósito parcial. A vazão calculada na calibração foi de 4,98 L.min ${ }^{-1}$. As pulverizações iniciaram-se antes do início da epidemia, no início da brotação, e foram direcionadas para as plantas, aplicando-se aproximadamente $25 \mathrm{~L}$ de volume de calda por tratamento. As parcelas de cada tratamento, no controle da ferrugem, receberam oito aplicações de fungicida, com intervalos de 15-20 dias, entre janeiro e maio de cada ano. Para furo-de-bala, foram 13 pulverizações entre outubro e abril, no mesmo intervalo utilizado para ferrugem.

A avaliação da eficiência dos fungicidas foi realizada com base na incidência e severidade das doenças, nas folhas e na porcentagem de desfolha, de janeiro a maio de cada safra, em intervalos que variavam entre 12 a 15 dias, dependendo das condições climáticas.

Para a avaliação das duas doenças foliares, foi marcado aleatoriamente um ramo misto por pernada, a uma altura aproximada de 1,70 m do chão. Avaliaram-se a incidência, pelo número de folhas com sintomas, e a severidade, pela porcentagem de área foliar lesionada, a qual foi realizada com o auxílio de escala diagramática para ferrugem (PERETTO; SILVA, 2002) e furo-de-bala (CHALLIOL et al., 2006). A desfolha causada pela ferrugem foi feita pela diferença no número total de folhas entre a primeira e a última avaliação.

Os dados foram analisados estatisticamente por Anova e teste de comparação de médias, ao nível de 5\% de significância, com auxílio do programa Sasm-Agri (CANTERI et al., 2001). Foram plotadas curvas de progresso da incidência de ferrugem, a partir da média dos valores obtidos das repetições, e a incidência, em função do tempo, foi analisada por meio de regressões não lineares, utilizando o software Statistica 6.0 (STATSOFT; TULSA, 2001). Para a seleção do modelo, foi considerado o maior valor do coeficiente de determinação $\left(\mathrm{R}^{2}\right)$.

\section{RESULTADOS E DISCUSSÃO}

A epidemia de ferrugem em Campo Largo iniciou-se em março e alcançou o valor máximo em maio, no tratamento-testemunha. Em Araucária, a doença teve início em janeiro, chegando a março com $100 \%$ de incidência na testemunha (Figura 1). Para furo-de-bala, a incidência máxima foi de $38 \%$, bem inferior ao já observado em safras anteriores, quando a incidência chegou a $90 \%$ das folhas (CHALLIOL et al., 2006). Esse fato pode ser explicado, em parte, principalmente pelo controle da sobrevivência do fungo nos cancros, realizado nos meses de inverno. O mesmo não ocorre com a ferrugem, quando todo ano o inóculo retoma o seu potencial, vindo também de áreas próximas onde 
o controle não tenha sido tão eficiente.

$\mathrm{O}$ ajuste dos dados de progresso da ferrugem no tratamento-testemunha, considerando a incidência da doença em proporção obtida com o modelo logístico $y=1 /\left(1+\left(\left(1 / y_{0}\right)-1\right) * \exp \left(-r^{*} x\right)\right)$. A taxa de progresso $(\mathrm{r})$ e o inóculo inicial $\left(\mathrm{y}_{0}\right)$ estimados foram 0,06 e 0,004 em 2004/2005; 0,09 e 0,02 em $20005 / 2006$, respectivamente. Não houve diferença significativa com relação à taxa de progresso da doença (r) e inóculo inicial para os dois anos avaliados (Figura 1). Entretanto, o que se observa de diferente entre as áreas é o período de início da epidemia, provavelmente influenciado pelo manejo da área em anos anteriores. Soto-Estrada e Adaskaveg (2004), na California/USA, avaliando o progresso da ferrugem em diferentes cultivares e locais, também não verificaram diferenças significativas entre as taxas durante os anos avaliados.

A severidade, no geral, foi baixa entre os tratamentos, nos dois anos avaliados, tanto para ferrugem como para furo-de-bala, não passando de 4\% (média das folhas no ramo) da área foliar afetada. Na primeira safra, a testemunha atingiu severidade de 3,05; e no ano seguinte, $3,42 \%$, diferindo significativamente dos outros tratamentos com produtos para controle da ferrugem; já para furo-de-bala, não houve diferença entre os produtos avaliados e a testemunha. Os fungicidas avaliados não deferiram significativamente nos dois anos para severidade das doenças (Tabela 1).

Quanto ao clorotalonil, Soto-Estrada et al.
(2003) verificaram a eficiência desse produto como um protetor foliar contra a ferrugem do pessegueiro na Califórnia (USA). Mueller et al. (2005) recomendaram esse fungicida no controle da severidade de cinco espécies de ferrugem do gênero Puccinia e uma espécie do gênero Pucciniastrum, em plantas ornamentais. Os mesmos autores testaram tiofanato-metílico que não se mostrou eficiente para esses patógenos.

Os baixos índices de severidade observados neste trabalho podem estar relacionados à temperatura elevada $\left(26\right.$ a $\left.32^{\circ} \mathrm{C}\right)$ e à precipitação pluviométrica (55 a $176 \mathrm{~mm}$ ) ocorrida de maneira irregular e muito intensa, durante o verão. Segundo Ellison et al. (1988), a temperatura é o principal fator que afeta a viabilidade do uredósporo, sendo bem críticas entre 25 e $30{ }^{\circ} \mathrm{C}$. De acordo com Martins e Amorim (1999), o intervalo de temperatura ótimo para que ocorra a infecção de ferrugem (T. discolor) é de 18 a $23{ }^{\circ} \mathrm{C}$, com um mínimo de quatro horas de molhamento foliar.

Em relação à desfolha, o clorotalonil diferenciou-se estatisticamente dos produtos avaliados, e o tiofanato metílico diferiu significativamente da testemunha, para ferrugem (Tabela 1). A desfolha é uma variável muito importante por ter relação direta com o dano (ALVES et al., 2008), sendo essenciais futuras pesquisas em diferentes regiões e pressão de inóculo, avaliando a ação dos ingredientes ativos sobre a manutenção das folhas.

TABELA 1 - Incidência, severidade e desfolha (\%) causadas pela ferrugem (T. discolor) e furo-de-bala ( $W$. carpophilus) em pessegueiro (Prunus persica) "Chimarrita", tratados com diferentes fungicidas. Safra2004/2005 e 2005/2006.

\begin{tabular}{lcccccccc}
\hline & \multicolumn{9}{c}{ Ferrugem } & \multicolumn{2}{c}{ Furo-de-Bala } \\
\hline Tratamentos & $2004 / 2005$ & $2005 / 2006$ & $2004 / 2005$ & $2005 / 2006$ & $2004 / 2005$ & $2005 / 2006$ & $2005 / 2006$ \\
\cline { 2 - 9 } & \multicolumn{2}{c}{ Incidência } & \multicolumn{2}{c}{ Severidade } & \multicolumn{2}{c}{ Desfolha } & Incidência & Severidade \\
\hline Testemunha & $62 \mathrm{a}$ & $94 \mathrm{a}$ & $3,05 \mathrm{a}$ & $3,42 \mathrm{a}$ & $37 \mathrm{a}$ & $62 \mathrm{a}$ & $37 \mathrm{a}$ & $0,36 \mathrm{~ns}$ \\
Mancozebe & $26 \mathrm{~b}$ & $58 \mathrm{ab}$ & $0,65 \mathrm{~b}$ & $1,4 \mathrm{~b}$ & $19 \mathrm{a}$ & $22 \mathrm{~b}$ & $24 \mathrm{~b}$ & 0,2 \\
Chorotalonil & $0,3 \mathrm{c}$ & $17 \mathrm{~b}$ & $0,02 \mathrm{~b}$ & $0,62 \mathrm{~b}$ & $12 \mathrm{a}$ & $2 \mathrm{~b}$ & $38 \mathrm{a}$ & 0,29 \\
Tiof.metílico & $19 \mathrm{~b}$ & $60 \mathrm{ab}$ & $0,55 \mathrm{~b}$ & $1,3 \mathrm{~b}$ & $11 \mathrm{a}$ & $11 \mathrm{~b}$ & $22 \mathrm{~b}$ & 0,15 \\
\hline
\end{tabular}

Médias seguidas pela mesma letra, nas colunas, não diferem entre si, pelo teste de Tukey (5\%), Ingredientes ativos: testemunha (sem pulverização), mancozebe (160g i.a./100L de água), clorotalonil (127,5g i.a./100L) e tiofanato metílico (51g i.a./100L). Cada valor representa média de oito ramos, em quatro repetições. 

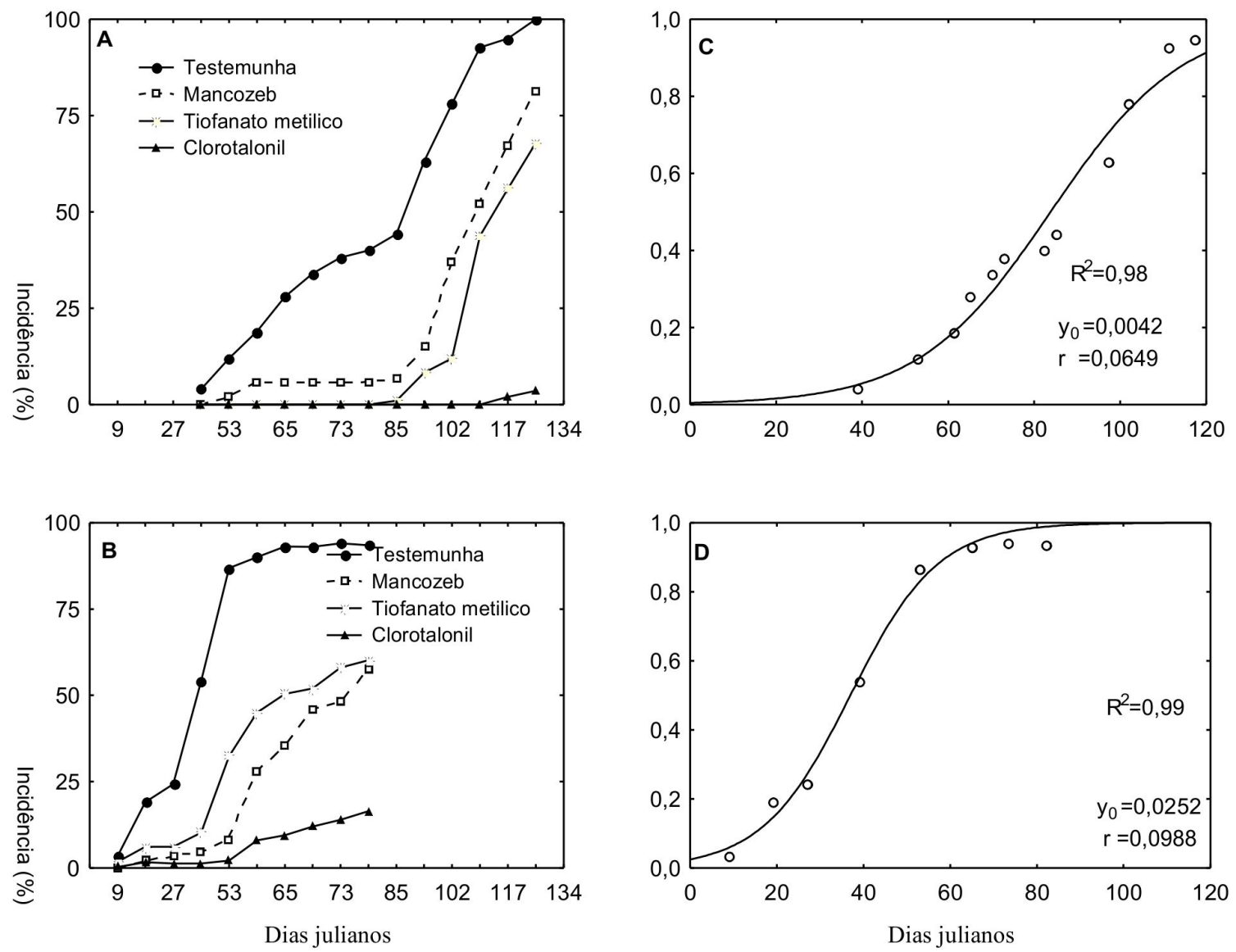

FIGURA 1 - Progresso da ferrugem do pessegueiro $(P$. persica $)$ cv. Chimarrita, medido pela incidência de ferrugem ( $T$. discolor) nas folhas de pomares, nos municípios de Campo Largo-PR em 2004/2005(A), e de Araucária-PR em 2005/2006 (B). Incidência da ferrugem em função do tempo no tratamento-testemunha, utilizando modelo logístico 2004/2005(C) e 2005/2006, (D), em que: $\mathrm{y}_{0}=$ inóculo inicial; $\mathrm{r}=$ taxa de progresso da doença, e $\mathrm{R}^{2}=$ coeficiente de determinação. Tratamentos: testemunha (sem pulverização), mancozeba ( $160 \mathrm{~g}$ i.a./100L de água), tiofanato metílico (51g i.a./100L) e clorotalonil(127,5g i.a./100L).

\section{CONCLUSÕES}

1. Foi evidenciado para a ferrugem o e furo-de-bala comportamento semelhante no progresso entre os pomares avaliados nos municípios da região metropolitana de Curitiba.

2. O ingrediente ativo clorotalonil reduziu a intensidade da ferrugem e a desfolha em folhas de pessegueiro, mas não foi eficiente no controle do furo-de-bala.

3. Os ingredientes ativos mancozebe e tiofanato metílico reduziram a incidência de furo-de-bala e a severidade da ferrugem.

\section{REFERÊNCIAS}

ADASKAVEG, J. E.; SOTO-ESTRADA, A.; FORSTER, H.; THOMPSON, D.; HASEY, J.; MANJI, B.T.; TEVIOTDALE, B. Peach rust caused by Tranzschelia discolor in Califórnia. Disponível em: $<$ http://www.anrcatalog.ucdavis.edu/pdf/8011.pdf $>$. Acesso em: 17 dez. 2009.

ALVES, G.; MAY-DE MIO, L.L.; ZANETTE, F.; OLIVEIRA, M.C. Ferrugem do pessegueiro e seu efeito na desfolha e na concentração de carboidratos em ramos e gemas. Tropical Plant Pathology, Brasília, v. 33, n.5, p.370-376, 2008. 
ALVES, G.; MAY-DE MIO, L.L. Efeito da desfolha causada pela ferrugem na floração e produtividade do pessegueiro. Revista Brasileira de Fruticultura, Jaboticabal, v.30, n.4, p.907-912, 2008.

CANTERI, M.G.; ALTHAUS, R.A.; VIRGENS F ${ }^{\circ}$, J.S.; GIGLIOTI, E.A.; GODOY, C.V. SASM-Agri: sistema para análise e separação de médias em experimentos agrícolas pelos métodos Scoft-Knott, Tukey e Duncan. Revista Brasileira de Agrocomputação, Ponta Grossa, v.1, n.2, p.18-24, 2001.

CHALLIOL, M.A.; MAY-DE MIO, L.L; CUQUEL, F.L.; MONTEIRO, L.; BEATRIZ MONTE SERRAT, B.; MOTTA, A.C.V.; RIBEIRO JR., P.J. Elaboração de escala diagramática para furo-de-bala e avaliação de doenças foliares em dois sistemas de produção de pessegueiro. Revista Brasileira de Fruticultura, Jaboticabal, v. 28, n. 3, p. 391-396, 2006.

ELLISON, P.J.; McFADYEN, L.M.; CULLIS, B.R. ; KABLE, P.F. Survival of dispersed urediniospores od tranzschelia discolor Fckl. (Traz. \& Litv.) on leaves of Prunus domestica L. cv. "Agen" in spring and summer in the Murrumbidge irrigation areas. Australian Journal of Agricultural Research, East Melbourne, n.39, p.847-56, 1988.
MARTINS, M.C.; AMORIM, L. Efeito do período de molhamento foliar em componentes monocíclicos da ferrugem do pessegueiro. Fitopatologia Brasileira, Brasília, v. 24, n.4 p.552-555, 1999.

MUELLER, D. S.; JEFFERS, S.N.; BUCK, J.W. Toxicity of fungicides to urediniospores of six fungi that occur on ornamental crops. Plant Disease, St. Paul, v.89, n.3, p. 255-261, 2005.

PERETTO, A.J.; SILVA, C.L. Guia de avaliação de ensaios: escalas diagramáticas e chaves descritivas. São Paulo: Departamento de Pesquisa e Desenvolvimento Hokko do Brasil Indústria Química e Agropecuária, 2002.

SOTO-ESTRADA, A.; FÖRSTER, H.; HASEY, J.; ADASKAVEG, J. E. New fungicides and application strategies based on inoculum and precipitation for managing stone fruit rust on peach in California. Plant Disease, St. Paul, v.87, n.9, p.1094-1101, 2003.

OTO-ESTRADA, A.; ADASKAVEG, J. E. Temporal and Quantitative Analyses of Stem Lesion Development and Foliar Disease Progression of Peach Rust in California. Phytopathology, St. Paul, v. 94, n. 1, 2004. 